\title{
Flow microfluorometric analysis of living spermatozoa stained with Hoechst 33342
}

\author{
K. D. Keeler, N. M. Mackenzie and D. W. Dresser \\ National Institute for Medical Research, Mill Hill, London NW7 IAA, U.K.
}

\begin{abstract}
Summary. Bovine spermatozoa were stained with Hoechst 33342. The fluorescence distribution of stained spermatozoa was complex. Non-motile spermatozoa displayed a higher fluorescence than did motile spermatozoa. The fluorescence profile of the motile spermatozoa was bimodal. Sort and reanalysis, and orientation experiments suggested that there are two distinct populations of motile spermatozoa.
\end{abstract}

\section{Introduction}

Flow microfluorometry is a convenient method for measuring the DNA content of mammalian cells (Tobey \& Crissman, 1975). Spermatozoa, by virtue of their ease of collection from many species, their homogeneity and their haploidy, are particularly suitable for such studies (Van Dilla et al., 1977; Gledhill, Lake \& Dean, 1979). To date, the majority of studies of the DNA content of spermatozoa have been carried out using fixed material stained with fluorochromes such as acridine orange, ethidium bromide, or mithramycin. Recently, the bisbenzimidazole dyes Hoechst 33258, Hoechst 33342, and DAPI (4',6'-diamidino-2-phenylindole) have been introduced as quantitative fluorescent stains for DNA. These dyes, although they bind tightly to DNA, do not intercalate into the molecule and hence are reputed not to disrupt its structure (Muller \& Gautier, 1975; Russell, Newman \& Williamson, 1975). These fluorochrome dyes are consequently capable of being used as quantitative vital stains for DNA: Hoechst 33258 and Hoechst 33342 have been used as vital stains to distinguish phases of the cell cycle (Lydon, Keeler \& Thomas, 1980).

Since spermatozoa are tail bearing and motile they orientate with their long axis along the line of flow in a flow microfluorometry system (Van Dilla et al., 1977). It has been concluded that an apparent bimodal DNA distribution in fixed acriflavine/Feulgen-stained bull sperm heads analysed in such a system, is due to an orientation artefact (Dean, Pinkel \& Mendelson, 1978), perhaps analogous to that previously described by Loken, Parks \& Herzenberg (1977) for the light scatter (size) artefact seen with chicken red blood cells (chicken RBC). Both of these artefacts can be by-passed or removed by the use of an appropriate nozzle which will control the orientation of flattened particles such as sperm heads or chicken RBC relative to the laser beam (Fulwyler, 1977; Stovel, Sweet \& Herzenberg, 1978; Dean et al., 1978). As an alternative approach, distribution artefacts can be tested by sorting the population into its separate components and then reanalysing them independently: if an artefact is involved, each reanalysed peak will give a bimodal peak similar to that observed originally.

In the study reported here we have used the bisbenzimidazole dye Hoechst 33342 as a vital fluorescent stain for DNA which allows spermatozoa to remain motile after analysis. The fluorescence distributions have been examined in detail using a commercially available fluorescence-activated cell sorter. 


\section{Materials and Methods}

\section{Spermatozoa}

Semen was collected, using the appropriate artificial vagina (First, 1971), from Friesian and Hereford bulls. Shortly after ejaculation, semen was added to 1-2 volumes of egg or milk medium at 20-22 ${ }^{\circ} \mathrm{C}$. Egg medium was kindly supplied by Mr J. Stables (Milk Marketing Board, Chase Farm, Bucks., U.K.). Milk medium was made according to the method of Almquist, Flipse \& Thacker (1954), which consists of centrifuging pasteurized milk at $2000 \mathrm{~g}$ for $10 \mathrm{~min}$, removing the cream, taking the underlying fat-free liquid from this slow speed spin and pelleting the milk solids by centrifugation at $48000 \mathrm{~g}$ for $30 \mathrm{~min}$. The clear supernatant is then heated at $92-96^{\circ} \mathrm{C}$ for $10 \mathrm{~min}$, followed by the addition of $0 \cdot 125 \mathrm{~g}$ D-fructose $/ \mathrm{ml}$ and antibiotics $\left(10^{4}\right.$ units penicillin $+10 \mathrm{mg}$ streptomycin sulphate per $100 \mathrm{ml}$ ) when cool. The spermatozoa were washed twice by centrifugation at $1000 \mathrm{~g}$ for $5 \mathrm{~min}$ followed by gentle resuspension of the pellet in sufficient fresh medium to give a concentration of $5 \times 10^{6}$ spermatozoa $/ \mathrm{ml}$. Cockerel semen was kindly supplied by Dr P. R. Woods (Houghton Poultry Research Station, Huntingdon, U.K.).

\section{Flow microfluorometric analysis}

Intact spermatozoa were stained with Hoechst 33342 in milk medium, at a concentration of 2 $\mu \mathrm{g} / \mathrm{ml}$ for bull and $5 \mu \mathrm{g} / \mathrm{ml}$ for cockerel, at room temperature for 2-3 $\mathrm{h}$. These dye concentrations were determined empirically from subjective assessment of optimal staining without overt cytotoxicity.

Flow microfluorometric analysis (Herzenberg, Sweet \& Herzenberg, 1976) was carried out using a Fluorescence Activated Cell Sorter (FACS II : Becton Dickinson Electronics Laboratories, Sunnyvale, California). The light source for the FACS was a 164-05 u.v.-enhanced argon-ion laser (Spectraphysics) which was operated at $20 \mathrm{~mW}$ in the u.v. Right-angle scatter of u.v. laser light was prevented from entering the fluorescence detector by a Wratten 2B filter. The FACS was calibrated in the u.v. using gluteraldehyde-fixed chicken RBC (Herzenberg \& Herzenberg, 1978).

Samples of spermatozoa were analysed and sorted at room temperature $\left(20-22^{\circ} \mathrm{C}\right)$ at a rate of up to 4000 cells/sec, except during orientation experiments (see below) in which the rate was reduced to $<800$ cells $/ \mathrm{sec}$. The sheath fluid was Dulbecco's phosphate-buffered saline ( $\mathrm{pH} 7 \cdot 2$; containing $\mathrm{Mg}^{2+}$ and $\mathrm{Ca}^{2+}$ ) but without stain.

A computer (Minc-11 Digital, MA, U.S.A.) linked to the FACS was used to calculate the total fluorescence (in arbitrary units) between channels 1 and 256 as follows:

$$
\text { Total fluorescence }=\sum_{1}^{256} \frac{\text { no. of cells in a channel } \times \text { channel no. }}{100}
$$

\section{Orientation}

Cells can be orientated into a single vertical plane at a predetermined angle to the laser beam by the method of Stovel et al. (1978). A (wedge shaped) sample injection tube, with faces set at $20^{\circ}$ to the axis of flow, was manufactured in the engineering department at NIMR. This device had the effect of making the (central) sample stream ribbon-shaped within the sheath stream. Since the velocity of the sheath is considerably higher than that of the sample stream, the latter is drawn into a thin ribbon and the flattened cells within this sample stream become orientated into the plane of the ribbon.

Extrapolating from maximal flow rates which allow successful orientation of chicken $\mathrm{RBC}$, we have estimated on the basis of cell (head) size and viscosity of the medium that successful orientation of spermatozoa should occur providing that the flow rate does not exceed $800 \mathrm{cells} / \mathrm{sec}$, when using a sample density of $5 \times 10^{6} / \mathrm{ml}$. 
When necessary, heads were removed from live spermatozoa in milk medium by ultrasonication for 5-10 $\mathrm{min}$ in a MSE ultrasonicator.

\section{Results}

A population of bull spermatozoa stained for a minimum of $2 \mathrm{~h}$ with Hoechst 33342 in milk medium shows a complex distribution of fluorescence intensity, which is illustrated in Text-fig. 1 . There are two pairs of peaks in the distribution, which have been labelled $\mathbf{A}$ and $\mathbf{B}$ respectively. When examined microscopically, cells from window B were non- (or only partly) motile, whereas spermatozoa sorted from window A showed active forward motility. The likelihood that the B peaks represent dead or moribund spermatozoa was tested by submitting a sample of stained spermatozoa to $56^{\circ} \mathrm{C}$ for $5 \mathrm{~min}$. This treatment left the spermatozoa totally immotile and when the fluorescence distribution of these immotile spermatozoa was examined (Text-fig. 1) the entire distribution was concentrated in the B peaks. A small peak seen between A and B in Text-fig. 1 may represent spermatozoa in a transitory state between $A$ and $B$ or the presence of a small percentage of diploid spermatozoa (Klasen \& Schmit, 1981).

Attention was concentrated on the A peaks of the fluorescence distribution of stained bull spermatozoa by running the FACS fluorescence gain at a higher setting (Text-fig. 2), so that the B peaks moved off-scale. The low and high peaks of the observed bimodal fluorescence distribution of the A peaks (AI and AII) contained approximately equal numbers of spermatozoa. The average fluorescence of spermatozoa in peak AII was approximately $30 \%$ higher than that in peak AI.

When cockerel spermatozoa were stained with $\mathrm{H} 33342$ the resulting fluorescence profile was quite different from that of bull spermatozoa (Text-fig. 3). The monophasic distribution of fluorescence may reflect either the homogametic nature of male birds or be due to the absence of an orientation artefact in the cylindrically headed cockerel spermatozoa.

The bimodal fluorescence distribution of bull spermatozoa may be due to a machine artefact, analogous to that observed for light scatter (size) analysis of chicken RBC (Loken et al., 1977), and therefore may not necessarily reflect underlying biological or physiological differences. An investigation into the nature of the observed bimodality was carried out by an analysis-sortreanalysis of stained spermatozoa and by the use of an 'orientating' nozzle.

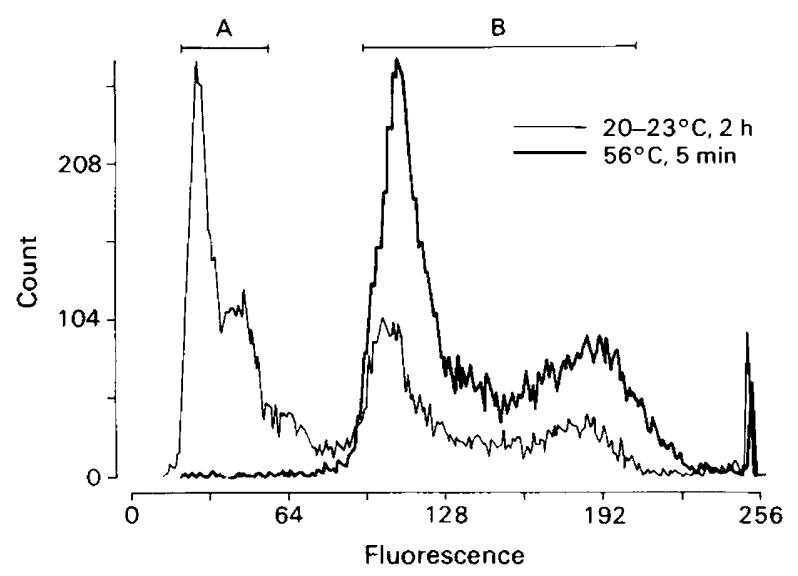

Text-fig. 1. The distribution of fluorescence of bull spermatozoa stained with Hoechst 33342 $(2 \mu \mathrm{g} / \mathrm{ml})$, showing the A and B peaks. Data are given for spermatozoa maintained in milk medium at ambient temperature $\left(20-23^{\circ} \mathrm{C}\right)$ for $2 \mathrm{~h}$ and those killed by being heated to $56^{\circ} \mathrm{C}$ for $5 \mathrm{~min}$. 




Text-fig. 2. As in Text-fig. 1, but with the machine run at a higher gain setting to remove the B peaks, and magnify the bimodality of peaks AI (low fluorescence) and AII.

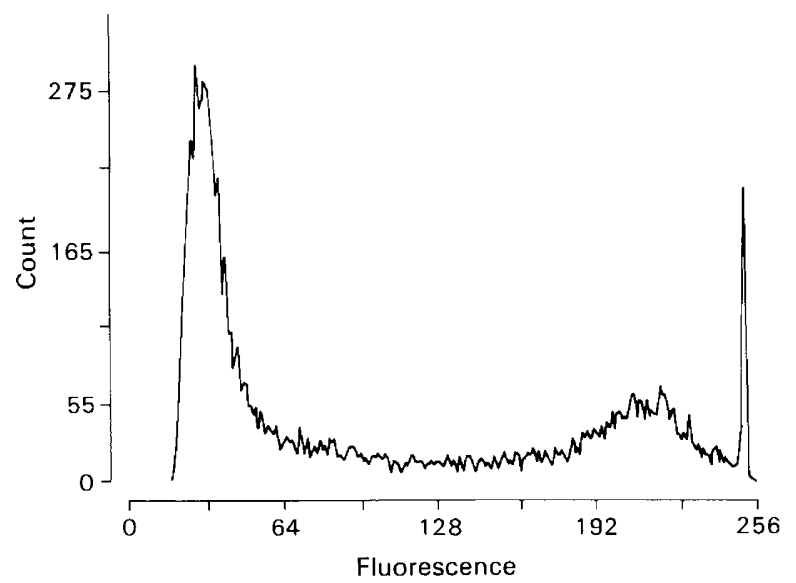

Text-fig. 3. Distribution of cockerel spermatozoa stained $(\sim 0.5 \times 4 \mu \mathrm{m}$ heads, $18 \mu \mathrm{m}$ tails $)$ with Hoechst $33342(5 \mu \mathrm{g} / \mathrm{ml})$ in egg medium.

First, the living Hoechst 33342-stained bull spermatozoa with a fluorescence distribution similar to that shown in Text-fig. 2, were physically separated (sorted) into an AI and AII population. Each separated population was then reanalysed and the respective fluorescence distributions are shown in Text-fig. 4. Although the peaks were not clearly unimodal, the spermatozoa from the high AII fraction had a higher overall fluorescence than those from AI as would be expected if the spermatozoa in peak AI were from a population different from that of those in peak AII. The low fluorescent peak appearing at approximately channel 30 for both populations in Text-fig. 4 was due to spermatozoa from which the H33342 had leached. Fixation of spermatozoa with buffered formal-saline ( $\mathrm{pH} \mathrm{7.4)} \mathrm{before} \mathrm{or} \mathrm{after} \mathrm{staining} \mathrm{or} \mathrm{after} \mathrm{they} \mathrm{had} \mathrm{been}$ sorted failed to reduce the leakage of dye. In 17 experiments in which the spermatozoa in peaks AI and AII were separated, the total fluorescence intensity of the reanalysed AII population was $15 \cdot 6 \pm$ $2.9 \%$ greater than that of the AI population. For a comparison, the same experiment was performed using chicken RBC. It is known that the apparent bimodal size distribution of the chicken RBC is an artefact related to the orientation of individual cells to the laser beam. When the chicken RBC 


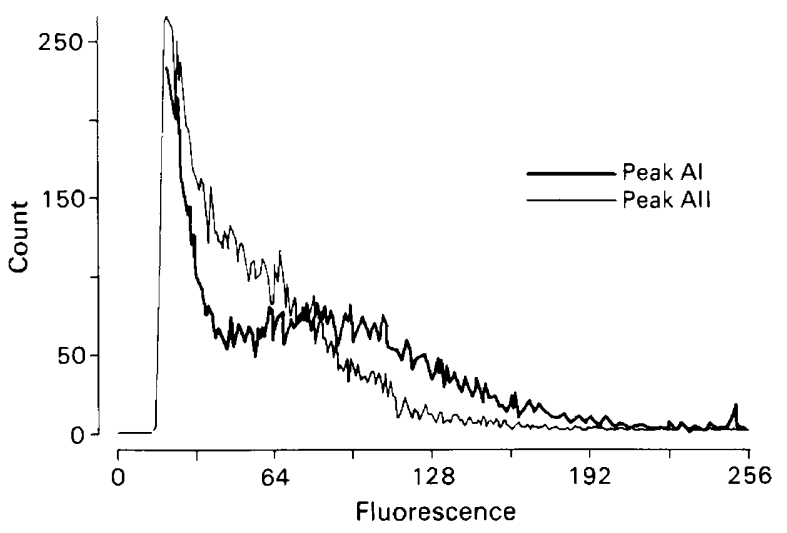

Text-fig. 4. High and low fluorescence peaks (AI and AII) of bull spermatozoa were separated according to the window settings indicated in Text-fig. 2 and reanalysed separately.

were sorted into two peaks on the basis of scatter, each separated peak gave the same bimodal distribution as the original, unsorted, material when reanalysed.

Second, an orientation nozzle similar to that originally developed by Stovel et al. (1978) was used to analyse bull spermatozoa. The efficiency of the nozzle was tested using a light-scatter analysis of chicken RBC (1200 cells/sec). The scatter distribution of chicken RBC (Text-fig. 5a) was affected by orientating the cells with their edges parallel to or at right angles to the laser beam. A similar effect was observed when sperm heads were passed through the orientating nozzle and the effect on the fluorescence profile examined. Although bull spermatzoa have flattened heads, they did not display a biphasic scatter (size) profile similar to that seen when analysing chicken RBC. Nevertheless, the heads of bull spermatozoa could be positively orientated, since the resulting fluorescence profiles were monophasic and did not overlap (Text-fig. 5b). In contrast, the bimodal fluorescence distribution of intact bull spermatozoa stained with Hoechst 33342 was not altered by rotation of the nozzle (Text-fig. 5c). The percentage of cells within each peak is shown in Table 1.

\section{Discussion}

Bull spermatozoa stained with Hoechst 33342 in milk or egg medium show a complex profile of fluorescence when analysed on the FACS. The observed fluorescence distribution of particles the size of spermatozoa ( $\sim 2 \times 5 \times 10 \mu \mathrm{m}$ head, $40 \mu \mathrm{m}$ tail) can be divided into three main areas: (1) unstained material, (2) a pair of highly fluorescent peaks (B) shown to consist of dead or moribund spermatozoa, and (3) a pair of peaks (AI and AII) with intermediate fluorescerice, which consist of spermatozoa with normal forward motility. We have concentrated our attention on peaks AI and AII.

An increased staining of non-viable cells by Hoechst 33342 similar to that seen here for bovine spermatozoa has previously been reported for dead or dying lymphocytes stained with the same dye (Szabo, Kiss \& Damjanovich, 1981). Szabo et al. (1981) suggested that the increased uptake of stain was due to a breakdown of the integrity of the cell membrane at cell death. This may be the mechanism responsible for the observed increase in flourescence of dead spermatozoa although it is possible that the normally tightly packed DNA in the nucleus becomes disorganized and this contributes to the increased staining. However, preliminary fluorometric studies (unpublished results) suggest that a considerable increase in the fluorescence intensity of Hoechst 33342 occurs as the $\mathrm{pH}$ decreases, irrespective of whether the dye is bound to DNA, protein or is free in solution. This observation suggests that the $\mathbf{B}$ peaks may arise because of increased nuclear acidity at death. 

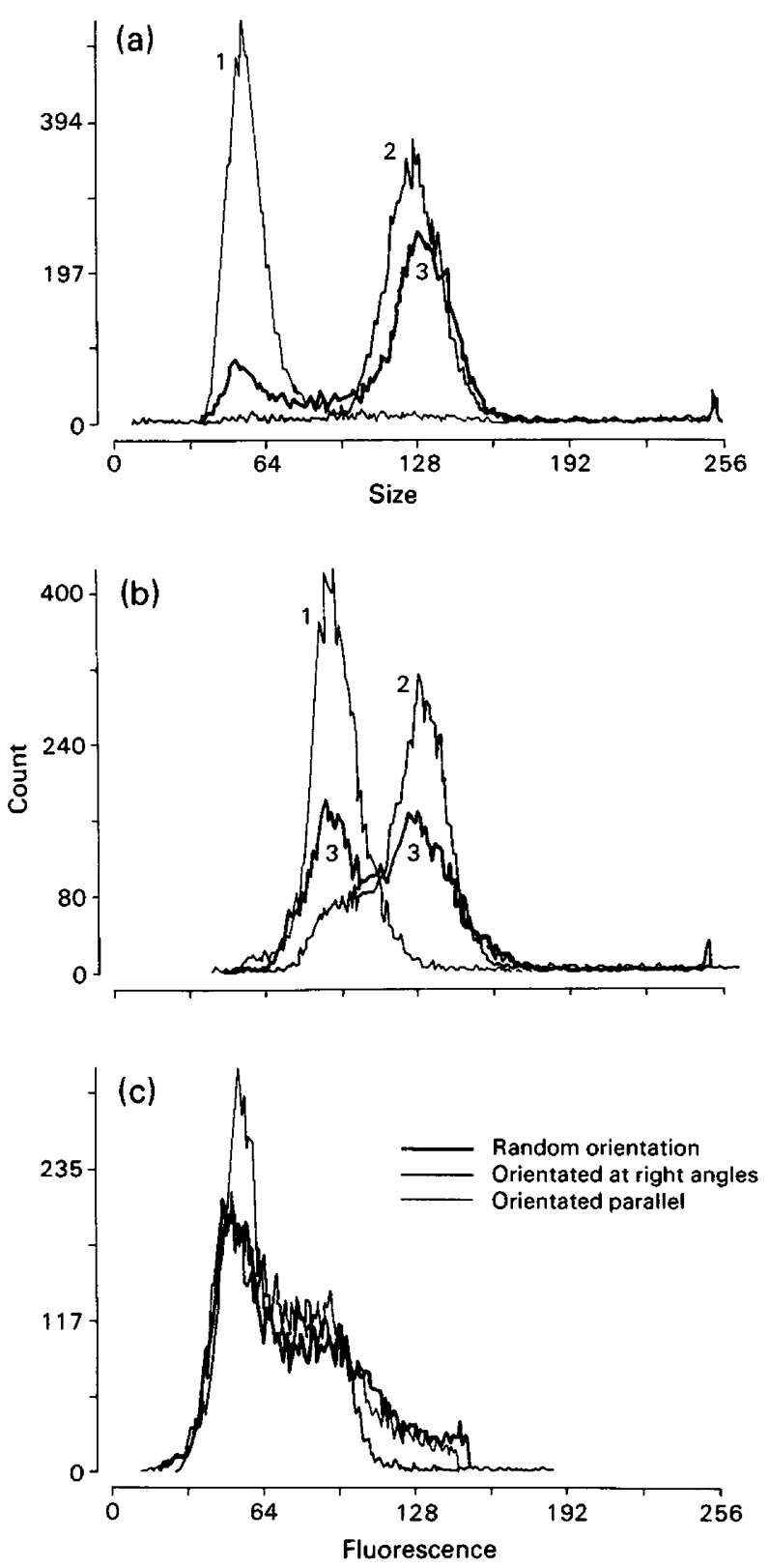

Text-fig. 5. Results using an orientating nozzle for (a) chicken RBC and (b, c) bull spermatozoa. In (a) peak 1 was obtained when the sample ribbon was parallel to the laser beam; peak 2 was obtained when the sample ribbon was at right angles to the laser beam, and peak 3 for randomly orientated cells. In (b) peak 1 was obtained when the heads of the spermatozoa were orientated edge on with respect to the laser beam and peak 2 when the sample was rotated through $90^{\circ}$ in the axis of flow (laser beam intersecting the broad side of head); randomly orientated cells are indicated by 3 . In (c) the bimodal distribution of fluorescence intensity of intact Hoechst 33342stained bull spermatozoa was not affected by altering the orientation of the sample ribbon: the distributions of randomly orientated and orientated cells overlapped. 
Table 1. Summary of the effect of an orientating nozzle on FACS analysis of chicken RBC (size) and bull spermatozoa (fluorescence) compared to non-orientated cells

\begin{tabular}{|c|c|c|c|c|c|c|}
\hline \multirow{3}{*}{$\begin{array}{l}\text { Orientation of } \\
\text { cell to } \\
\text { laser beam }\end{array}$} & \multirow{2}{*}{\multicolumn{2}{|c|}{$\begin{array}{c}\text { Chicken } \\
\text { RBC }\end{array}$}} & \multicolumn{4}{|c|}{ Bull spermatozoa } \\
\hline & & & \multicolumn{2}{|c|}{ Heads } & \multicolumn{2}{|c|}{ Intact } \\
\hline & $\begin{array}{l}\text { Low } \\
\text { Peak }\end{array}$ & $\begin{array}{l}\text { High } \\
\text { Peak }\end{array}$ & $\begin{array}{l}\text { Low } \\
\text { Peak }\end{array}$ & $\begin{array}{l}\text { High } \\
\text { Peak }\end{array}$ & $\begin{array}{l}\text { Low } \\
\text { Peak }\end{array}$ & $\begin{array}{l}\text { High } \\
\text { Peak }\end{array}$ \\
\hline $\begin{array}{l}\text { Random } \\
\text { (normal nozzle) }\end{array}$ & 22 & 78 & 43 & 57 & 51 & 49 \\
\hline Narrow side & 94 & 6 & 90 & 10 & 59 & 41 \\
\hline Broad side & 3 & 97 & 22 & 78 & 51 & 49 \\
\hline
\end{tabular}

The values are the no. of cells in each peak of the distribution expressed as a $\%$ of the total.

The bimodal distribution observed in the Hoechst 33342 staining of viable spermatozoa (peaks A) could be a machine orientation artefact or a consequence of the biologically different kinds of spermatozoa in the normal ejaculate. We have tested the hypothesis that bimodality is artefactual, in three ways: (1) By comparison of the fluorescence profiles of mammalian and bird spermatozoa which are heterogametic and homogametic respectively. The cockerel spermatozoa had a unimodal distribution (Text-fig. 3). (2) Spermatozoa were analysed using an orientating nozzle. The technique was shown to be effective for chicken RBC (Stovel et al., 1978). Text-figure 5 illustrates that although the heads of spermatozoa can be orientated the bimodal fluorescence distribution of Hoechst 33342-stained intact live spermatozoa is apparently independent of the orientation of the sperm heads around their long axis. Alternatively, as Dean et al. (1978) believe, tail bearing spermatozoa may not be orientated in the stream by an orientating nozzle. (3) We separated viable bull spermatozoa into peaks AI and AII and then analysed the two populations. Peaks AI and AII appear to retain much of their integrity (Text-fig. 4); although not clearly unimodal, they are of predictable fluorescence in that spermatozoa separated from peak AII fluoresce more brightly than those from AI : a difference which averages at about $15 \%$. If bimodality had been an artefact we would have expected the separated population to have identical (bimodal) distributions. This principle has been demonstrated using the chicken RBC size (light-scatter) artefact.

On the strength of the results above we conclude that the observed bimodality of fluorescence distribution is not, on the whole, artefactual and indicates the presence of two physiologically or biologically different subpopulations of viable spermatozoa. The subpopulations (AI and AII) may reflect spermatozoa at distinct stages of late maturation or the difference between $\mathrm{X}$ - and $\mathrm{Y}$ chromosome bearing spermatozoa. However, the degree of difference represented by the apparent amount of stain taken up by spermatozoa in the two populations is nearer $15-30 \%$ than the $2-3 \%$ estimated from the differences between autosomes plus X compared to autosomes plus $\mathrm{Y}$. Although Hoechst 33342 uptake is generally assumed to be a quantitative measure of DNA content (Lydon et al., 1980), it is possible that staining also reflects other factors such as the packaging of the DNA or the amount of heterochromatin present.

We thank Professor D. A. Noakes and his staff at the Department of Veterinary Obstetrics and Gynaecology, Royal Veterinary College, Brookmans Park, Hertfordshire, for supplying the bull semen, and Mr J. Stables (Milk Marketing Board, Chase Farm, Little Harwood, Bucks) for his help and advice. 


\section{References}

Almquist, J.O., Flipse, R.J. \& Thacker, D.L. (1954) Diluters of bovine semen. IV. Fertility of bovine spermatozoa in heated homogenized milk and skimmed milk. J. Dairy Sci. 37, 1303-1304.

Dean, P.N., Pinkel, D. \& Mendelson, M.L. (1978) Hydrodynamic orientation of sperm heads for flow cytometry. Biophys. J. 23, 7-13.

First, N.L. (1971) Collection and preservation of sperm. In Methods in Mammalian Embryology, pp. 15-35. Ed. J. C. Daniel, Jr. Freeman, San Francisco.

Fulwyler, M.J. (1977) Hydrodynamic orientation of cells. J. Histochem. Cytochem. 25, 781-783.

Gledhill, B.L., Lake, S. \& Dean, P.N. (1979) Flow cytometry and sorting of sperm and other male germ cells. In Flow Cytometry and Sorting, pp. 471-485. Eds M. R. Melamed, P. F. Mullaney \& M. L. Mendelsohn. Wiley, New York.

Herzenberg, L.A. \& Herzenberg, L.A. (1978) Analysis and separation using the fluorescence activated cell sorter. In Handbook of Experimental Immunology, 3rd edn, pp. 22.1-22.21. Ed. D. M. Weir. Blackwell Scientific Publications, Oxford.

Herzenberg, L.A., Sweet, R.G. \& Herzenberg, L.A. (1976) Flourescence activated cell sorting. Sci. Am. 234, $108-117$.

Klasen, M. \& Schmit, M. (1981) An improved method for $\mathrm{Y}$ body identification and confirmation of a high incidence of YY sperm nuclei. Hum. Genet. 58, 156 161 .
Loken, M.R., Parks, D.R. \& Herzenberg, L.A. (1977) Identification of cell asymmetry and orientation by light scattering. J. Histochem. Cytochem. 25, 790-795.

Lydon, M.J., Keeler, K.D. \& Thomas, D.B. (1980) Vital DNA staining and cell sorting by flow micro-fluorometry. J. cell. Physiol. 102, 175-181.

Muller, W. \& Gautier, F. (1975) Interaction of heteroaromatic compounds with nucleic acid A-T specific non-intercalating DNA ligands. Eur. J. Biochem. 54, 385-394.

Russell, W.C., Newman, C. \& Williamson, D.H. (1975) A simple cytochemical technique for demonstration of DNA in cells infected with mycoplasma and viruses. Nature, Lond. 253, 461-462.

Stovel, R.T., Sweet, R.G. \& Herzenberg, L.A. (1978) A means for orienting flat cells in flow systems. Biophys. J. 23, 1-5.

Szabo, G., Jr, Kiss, A. \& Damjanovich, S. (1981) Flow cytometric analysis of the uptake of Hoechst 33342 dye by human lymphocytes. Cytometry 2, 20-23.

Tobey, R.A. \& Crissman, H.A. (1975) Unique techniques for microfluorometry. Expl Cell Res. 93, 235-239.

Van Dilla, M.A., Gledhill, B.L., Lake, S., Dean, P.N., Gray, J.W., Kachel, V., Barlogie, B. \& Gohde, W. (1977) Measurement of mammalian sperm deoxyribonucleic acid by flow cytometry. Problems and approaches. J. Histochem. Cytochem. 25, 763-773.

Received 27 September 1982 